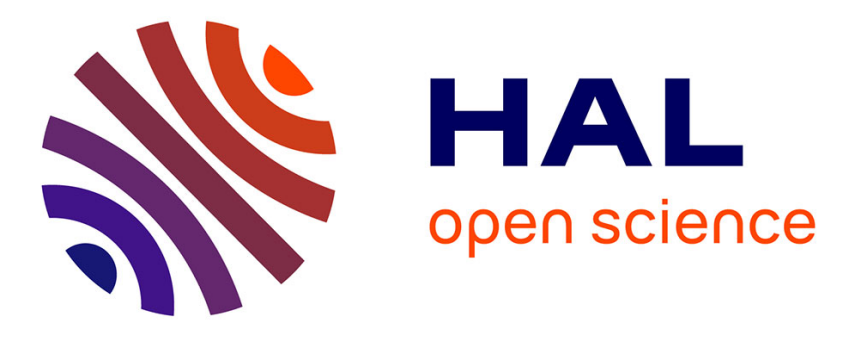

\title{
Self-propelling uneven Leidenfrost solids
}

Guillaume Dupeux, Tobias Baier, Vincent Bacot, Steffen Hardt, Christophe

Clanet, David Quéré

\section{To cite this version:}

Guillaume Dupeux, Tobias Baier, Vincent Bacot, Steffen Hardt, Christophe Clanet, et al.. Selfpropelling uneven Leidenfrost solids. Physics of Fluids, 2013, 25 (5), pp.051704. 10.1063/1.4807007 . hal-00996482

\section{HAL Id: hal-00996482 \\ https://hal-polytechnique.archives-ouvertes.fr/hal-00996482}

Submitted on 2 Jun 2014

HAL is a multi-disciplinary open access archive for the deposit and dissemination of scientific research documents, whether they are published or not. The documents may come from teaching and research institutions in France or abroad, or from public or private research centers.
L'archive ouverte pluridisciplinaire HAL, est destinée au dépôt et à la diffusion de documents scientifiques de niveau recherche, publiés ou non, émanant des établissements d'enseignement et de recherche français ou étrangers, des laboratoires publics ou privés. 


\section{AIP $\mid$ Physics of Fluids}

\section{Self-propelling uneven Leidenfrost solids}

Guillaume Dupeux, Tobias Baier, Vincent Bacot, Steffen Hardt, Christophe Clanet, and David Quéré

Citation: Physics of Fluids (1994-present) 25, 051704 (2013); doi: 10.1063/1.4807007

View online: http://dx.doi.org/10.1063/1.4807007

View Table of Contents: http://scitation.aip.org/content/aip/journal/pof2/25/5?ver=pdfcov

Published by the AIP Publishing

\section{Articles you may be interested in}

Two dimensional Leidenfrost droplets in a Hele-Shaw cell

Phys. Fluids 26, 032103 (2014); 10.1063/1.4867163

Curvature singularity and film-skating during drop impact

Phys. Fluids 23, 091701 (2011); 10.1063/1.3640028

Spontaneous mode-selection in the self-propelled motion of a solid/liquid composite driven by interfacial instability

J. Chem. Phys. 134, 114704 (2011); 10.1063/1.3567096

Breakup of diminutive Rayleigh jets

Phys. Fluids 22, 122003 (2010); 10.1063/1.3524533

Breakup of drops in a microfluidic T junction

Phys. Fluids 21, 023303 (2009); 10.1063/1.3078515

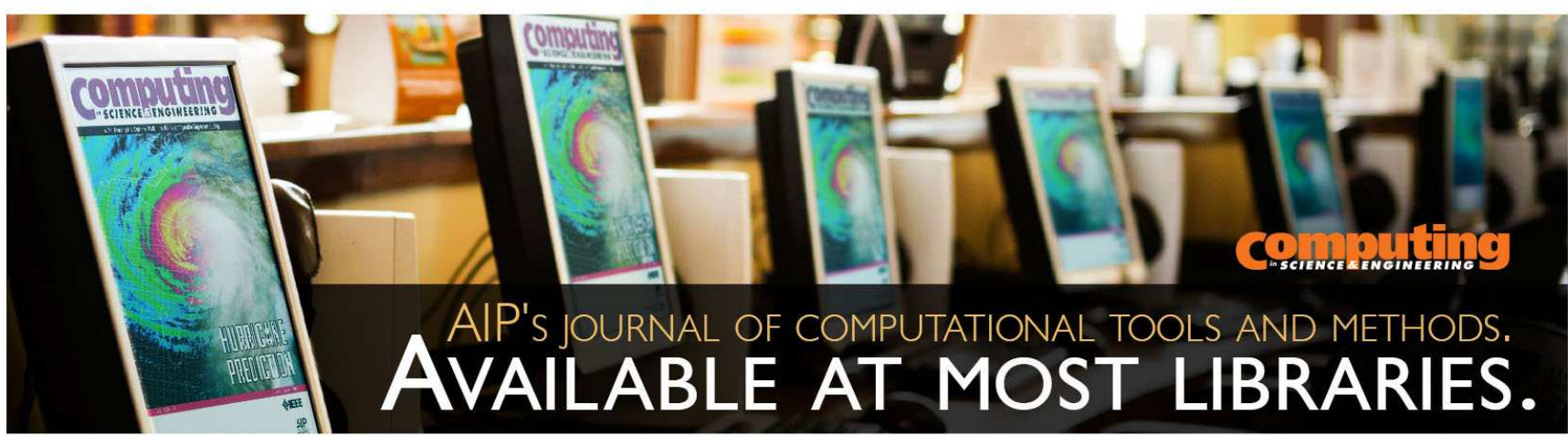




\title{
Self-propelling uneven Leidenfrost solids
}

\author{
Guillaume Dupeux, ${ }^{1,2}$ Tobias Baier, $^{3}$ Vincent Bacot, ${ }^{2}$ Steffen Hardt, ${ }^{3}$ \\ Christophe Clanet, ${ }^{1,2}$ and David Quéré ${ }^{1,2}$ \\ ${ }^{1}$ Physique et Mécanique des Milieux Hétérogènes, UMR 7636 du CNRS, ESPCI, \\ 75005 Paris, France \\ ${ }^{2}$ Ladhyx, UMR 7646 du CNRS, École Polytechnique, 91120 Palaiseau, France \\ ${ }^{3}$ Center of Smart Interfaces, TU Darmstadt, Darmstadt, Germany
}

(Received 30 November 2012; accepted 15 March 2013; published online 23 May 2013)

\begin{abstract}
Placed on a hot surface, a solid that sublimates at atmospheric pressure can levitate on a cushion of its own vapor. Discovered by Leidenfrost, this effect has mostly been studied with liquids. Whereas the shape of a droplet is determined by a competition between gravity, surface tension, and stress in the vapor layer, a solid does not deform. In this paper, we show experimentally and theoretically that asymmetric mass distributions in a Leidenfrost solid can lead to a non homogenous vapor layer in which the lubrication flow generates a lateral force able to propel the body. (c) 2013 AIP Publishing LLC. [http://dx.doi.org/10.1063/1.4807007]
\end{abstract}

Droplets, on a surface whose temperature is high above the boiling point of the liquid, levitate. In the Leidenfrost effect, ${ }^{1}$ the strong evaporation creates a cushion of vapor whose pressure can be high enough to sustain the drop. The absence of contact with the substrate reduces a lot of the friction, ${ }^{2}$ which generates bouncing ${ }^{3}$ and vibrations ${ }^{4}$ making the control of such drops problematic. ${ }^{5}$ Levitation and vapor production were exploited by Linke $e t$ al. who designed a substrate made of millimetric asymmetric teeth: a Leidenfrost drop placed on this ratchet gets propelled in a welldefined direction. ${ }^{6}$ The explanation for this effect has been recently debated. ${ }^{7-10}$ Linke suggested a viscous entrainment as responsible for the motion, which was confirmed by experiments and simulations where platelets of solid carbon dioxide were found to similarly self-propel on hot ratchets $^{7,9,10}$ (Figure 1). Solid carbon dioxide, also called dry ice, sublimates at $-78{ }^{\circ} \mathrm{C}$ : left on a hot plate, it floats in the Leidenfrost state. Since dry ice is not deformable, the geometry is made much simpler compared to a liquid, which helped to confirm that propulsion arises from a viscous drive by the underlying vapor flow. ${ }^{9,10}$

Unlike liquids, solids can be sculpted into different shapes. In this paper, we investigate experimentally and theoretically the behavior of uneven pieces of dry ice placed on horizontal smooth plates. To control the unevenness, we add a small weight on the side of a cylindrical platelet (Figure 2). The non-homogenous distribution of mass leads to non-uniform vapor layers, as sketched in Figure 3. The bottom of the dry ice is tilted by a small angle $\theta$, the vapor layer being thinner where the body is heavier. As a consequence, the platelet does not remain at rest, it self-propels in

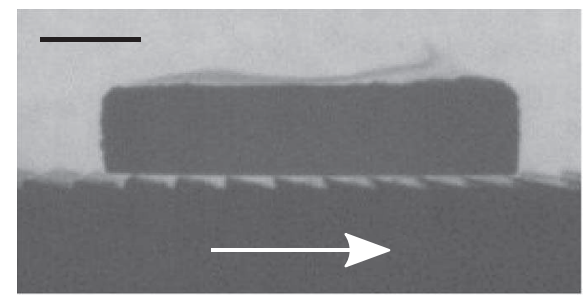

FIG. 1. A platelet of dry ice self-propels on a hot ratchet $\left(450^{\circ} \mathrm{C}\right)$. The white arrow shows the direction of propulsion. The bar indicates $3 \mathrm{~mm}$ (enhanced online). [URL: http://dx.doi.org/10.1063/1.4807007.1] 


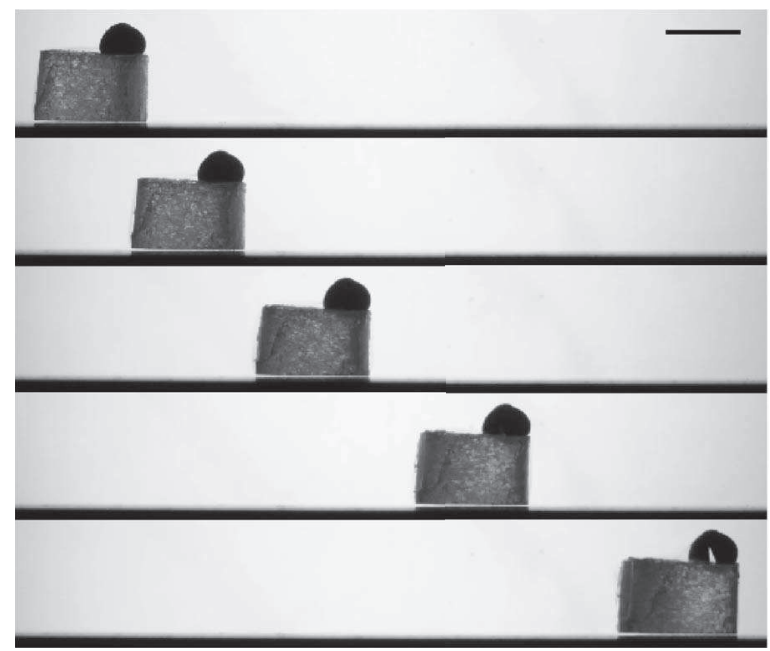

FIG. 2. A centimeter-size platelet of dry ice on a hot flat surface $\left(320^{\circ} \mathrm{C}\right)$ with a small weight $(m=0.54 \mathrm{~g})$ on the right side. The added mass tilts the platelet (by an angle $\theta=0.3^{\circ}$ ), so that the ice gets propelled (enhanced online). [URL: http://dx.doi.org/10.1063/1.4807007.2]. Two pictures are separated by $0.3 \mathrm{~s}$ and the scale represents $1 \mathrm{~cm}$. The asymmetry can also be made by sculpting the dry ice (enhanced online). [URL: http://dx.doi.org/10.1063/1.4807007.3]

the direction of the added weight. Geometrically, this system seems close to the one of axisymmetric platelets on ratchets: ${ }^{6-10}$ in both cases, the gap between the levitating solid and the substrate makes an angle (asymmetric platelet) or a series of angles (ratchet), which creates propulsion. However, the motion is directed toward the direction of closing angle for the asymmetric ice (Figure 2), whereas it takes place in the direction of opening angles over a ratchet (Figure 1).

The vapor flow exerts two forces on the platelet (Figure 3(b)): a viscous force $\boldsymbol{F}_{S}$, parallel to the bottom surface, and a pressure force $\boldsymbol{F}_{P}$, perpendicular to this surface. We expect $\boldsymbol{F}_{S}$ to be in the direction of opening angle, like for the ratchet where it is indeed the driving force. However, the bottom surface of the asymmetric platelet being tilted, the pressure force $\boldsymbol{F}_{P}$ also has a horizontal component $\theta F_{P}$ that acts in the direction of propulsion. If it exceeds the viscous contribution $\left(\theta F_{P}>F_{S}\right)$, the propulsion can indeed take place in the direction of closing angle $\theta$.

Experiments are performed with dry ice platelets of radius $R=7.6 \pm 0.4 \mathrm{~mm}$ and height $H=10.0 \pm 0.5 \mathrm{~mm}$ hovering over a flat aluminum surface brought to a temperature of $320^{\circ} \mathrm{C}$. We record the ice trajectory $x(t)$ with a high speed video camera on a distance of $15 \mathrm{~cm}$. Figure 4 shows the time-dependence of the velocity $v=x^{\prime}(t)$ for three platelets with different added masses $m$ of $0.21 \mathrm{~g}, 0.78 \mathrm{~g}$, and $1.24 \mathrm{~g}$. In comparison, the mass of the platelet is $M=2.8 \mathrm{~g}$. The velocity of a platelet is a linear function of time, from which we can derive a constant acceleration $x^{\prime \prime}$, and thus

(a)

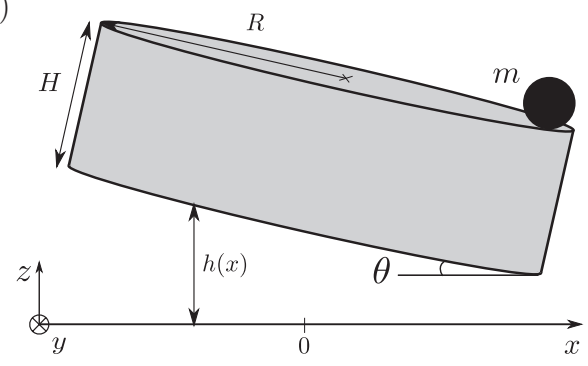

(b)

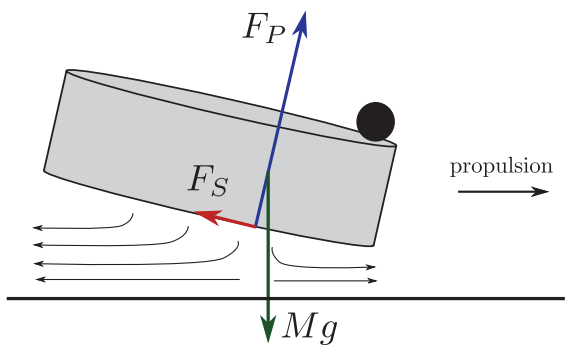

FIG. 3. (a) Sketch of the experiment: a platelet of dry ice of mass $M$ with an extra weight of mass $m$ on a side hovers above a flat surface. (b) Viscous force $\boldsymbol{F}_{S}$ and pressure force $\boldsymbol{F}_{P}$ applied by the vapor flow on the platelet. 


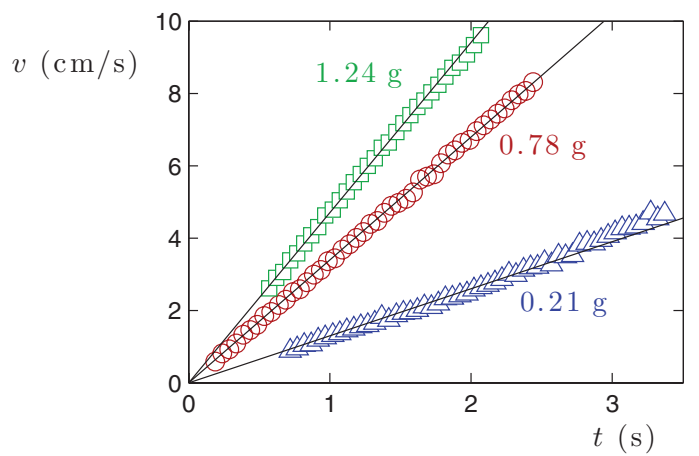

FIG. 4. Velocity $v$ as a function of time for a given platelet (of mass $M=2.8 \mathrm{~g}$ ) and different added weights (of respective masses $m=0.21 \mathrm{~g}, 0.78 \mathrm{~g}$, and $1.24 \mathrm{~g}$ ). The stronger the added mass, the more accelerated the platelet (enhanced online). [URL: http://dx.doi.org/10.1063/1.4807007.4]

deduce the propelling force $F=(M+m) x^{\prime \prime}$. We find forces on the order of $100 \mu \mathrm{N}$, which increase with the added weight.

In order to understand the origin of the propelling force, we focus on the flow in the vapor layer (Figure 3). Due to the added weight, the platelet makes an angle $\theta$ with the horizontal. Experimentally, $\theta$ is typically $0.01 \mathrm{rad}$ and it increases with $m$, always satisfying $\theta \ll 1$. Since the vapor layer is thin, with a thickness $h_{0}$ much smaller than $R\left(h_{0} \approx 100 \mu \mathrm{m}\right.$ and $\left.R=7.6 \mathrm{~mm}\right)$, the flow below a Leidenfrost solid can be described by a viscous flow in the lubrication approximation. ${ }^{9}$ The local flux conservation is written $\boldsymbol{\nabla} .(h \boldsymbol{U})=V$, where $\boldsymbol{U}(x, y)$ is the horizontal velocity averaged along the $z$-axis, $h(x)$ is the local thickness of the vapor layer $\left(h=h_{0}-\theta x\right)$, and $V(x)$ is the velocity of the vapor ejected by sublimation at the surface of the platelet. Conduction dominates the heat transfer, ${ }^{11}$ so that $V$ is equal to $(\kappa / \rho L)(\partial T / \partial z)$, where $T, \rho$, and $\kappa$ are, respectively, the temperature, the density, and the thermal conductivity of the vapor $\left(\rho=1 \mathrm{~kg} / \mathrm{m}^{3}, \kappa \approx 0.03 \mathrm{~W} / \mathrm{m} / \mathrm{K}\right)$, and $L$ is the latent heat of sublimation of dry ice $\left(L \approx 7.10^{5} \mathrm{~J} / \mathrm{kg}\right.$ ). Since $R \gg h$, we assume $\partial T / \partial z \approx \Delta T / h$, where $\Delta T$ is the temperature difference between the solid and the platelet $\left(\Delta T \approx 400^{\circ} \mathrm{C}\right)$. $\boldsymbol{U}$ is calculated assuming a Poiseuille law in the vapor layer: $\boldsymbol{U}=-\left(h^{2} / 12 \eta\right) \nabla p$, where $p(x, y)$ is the vapor pressure and $\eta$ its dynamic viscosity $\left(\eta \approx 2.10^{-5} \mathrm{~Pa}\right.$ s). This particular case of Darcy's law is obtained for a flow in a narrow gap, averaged over the perpendicular coordinate ( $z$-axis here). With the conservation of flux, it leads to the differential equation

$$
\nabla \cdot\left(h^{3} \nabla p\right)=-\frac{12 \eta \kappa \Delta T}{\rho L h}
$$

with the condition $p=0$ on the vapor layer boundary. $h(x)$ can be written $h_{0}(1-\varepsilon x)$ with $\varepsilon=R \theta / h_{0}$. Assuming $\varepsilon \ll 1$, we expand $p$ in $\varepsilon, p=p_{0}+\varepsilon p_{1}+\mathcal{O}\left(\varepsilon^{2}\right)$, and solve the two first orders in Eq. (1) (see the supplementary material for the whole calculation ${ }^{12}$ ). We calculate the pressure force $F_{P}$ and the shear force $F_{S}$ that are, respectively, normal and tangential to the bottom face of the platelet, as well as the moment of the pressure force around the $y$-axis, $\Gamma_{P}$. We obtain at first order:

$$
\begin{gathered}
F_{S}=-\int_{S} \eta\left(\frac{\partial u_{x}}{\partial z}\right)_{h} d S=-\frac{3 \pi}{4} \frac{\eta \kappa \Delta T R^{3}}{\rho L h_{0}^{3}} \varepsilon, \\
F_{P}=\int_{S} p d S=\frac{3 \pi}{2} \frac{\eta \kappa \Delta T R^{4}}{\rho L h_{0}^{4}}, \\
\Gamma_{P}=-\int_{S} x p d S=-\frac{11 \pi}{16} \frac{\eta \kappa \Delta T R^{5}}{\rho L h_{0}^{4}} \varepsilon
\end{gathered}
$$

where $S$ is the bottom surface of the platelet $\left(S=\left\{(x, y) \mid x^{2}+y^{2} \leq R^{2}\right\}\right)$. The projection of $F_{S}$ and $F_{P}$ on the vertical axis balances the weight: $F_{P} \cos \theta-F_{S} \sin \theta=(M+m) g$. Since we have $\theta \ll 1$ 

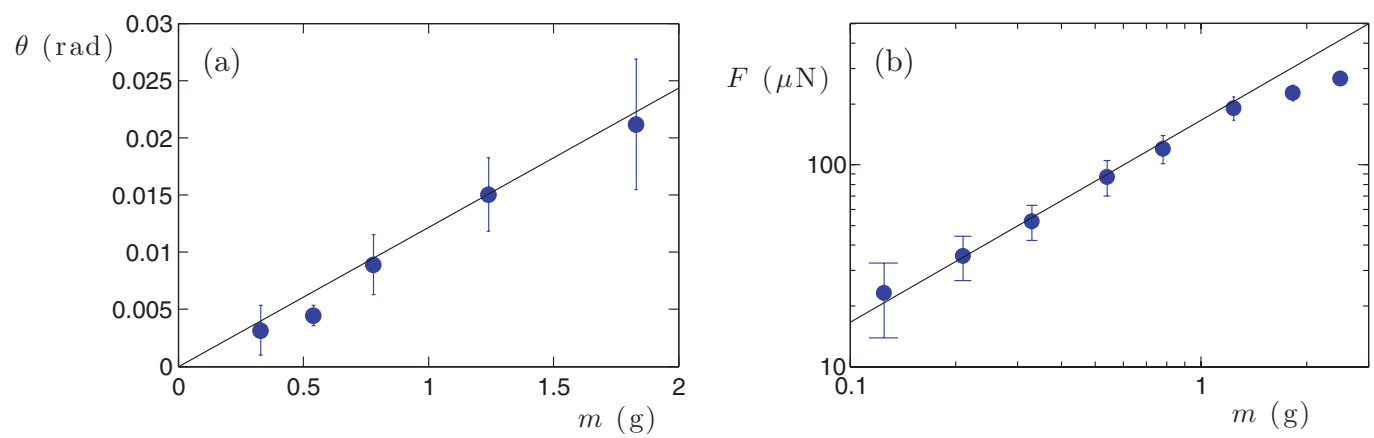

FIG. 5. (a) Measured angle $\theta$ as a function of the added mass $m$. The solid line represents Eq. (4), its slope is given by $24 /(11 M) \sqrt{b / R}=12 \mathrm{rad} / \mathrm{kg}$. Each point is an average on five measurements. (b) Measured driving force $F$ as a function of the added mass $m$. The solid line corresponds to Eq. (5). Each point is an average on ten measurements.

and $F_{S} \theta / F_{P} \sim \theta^{2}$, this balance leads to $F_{P}=(M+m) g$, which yields the mean thickness of the vapor layer,

$$
h_{0}=\sqrt{b R},
$$

where the distance $b$ is $\left[3 \eta \kappa \Delta T / 2 \rho \rho_{0} \operatorname{LgH}(1+m / M)\right]^{1 / 2}$, with $\rho_{0}$ the dry ice density $\left(\rho_{0}=1560 \mathrm{~kg} / \mathrm{m}^{3}\right)$ and $H$ the platelet height. As expected, we find the expression of the film thickness for an axisymmetric system. ${ }^{7}$ Using Eq. (3) and assuming the additional mass to sit right at the edge of the cylinder, the moment balance around the $y$-axis, $\Gamma_{P}+m g R=0$, leads to

$$
\theta=\frac{24}{11} \frac{m}{M} \sqrt{\frac{b}{R}}
$$

A first consequence of Eq. (4) is to show that our hypothesis $\varepsilon \ll 1$ is equivalent to $m \ll M$ $\left(\varepsilon=R \theta / h_{0} \sim m / M\right)$, roughly obeyed in our experiments. Then the expression of $b$ simplifies to $\left(3 \eta \kappa \Delta T / 2 \rho \rho_{0} L g H\right)^{1 / 2}$, which only depends on physical constants and on the platelet height $H$, with a typical value of $1 \mu \mathrm{m}$.

We finally project $F_{S}$ and $F_{P}$ on the horizontal axis to obtain the propelling force: $F=F_{S}$ $+\theta F_{P}$. Equations (2a) and (2b) show that both components of $F$ have the same scaling yet opposite directions. However, it is found that the contribution of the pressure $\theta F_{P}$ is twice the contribution of the shear $F_{S}$ (see the supplementary material for more details ${ }^{12}$ ). The propelling mechanism in this system is then different from the one in the ratchet, making ice move in the opposite direction towards the narrow side of the gap. On the whole, the propelling force can be written: $F=M g \theta / 2$. This very simple expression mixes the genuine driving force of the motion (the weight of the solid, which forces a vapor flow below) with its cause (the angle $\theta$, which quantifies the degree of asymmetry of the system). Together with Eq. (4), we obtain

$$
F=\frac{12}{11} m g \sqrt{\frac{b}{R}}
$$

These findings can be compared to experiments. First, Figure 5(a) shows the measured angle $\theta$ as a function of the added mass $m$. The platelets are the same as described previously. We take a picture of a moving platelet and we measure $\theta$ directly, after magnifying the pictures (each point is an average on five measurements). The solid line shows Eq. (4) calculated with the numerical values of the different parameters. The model is in good agreement with the experiments: the angle increases linearly with $m$ and Eq. (4) nicely fits the data without any adjustable parameter. Second, Figure 5(b) shows the force (deduced from the acceleration of the platelet) as a function of the added mass. The solid line is plotted using Eq. (5). Again, we observe a linear increase (the solid line has a 


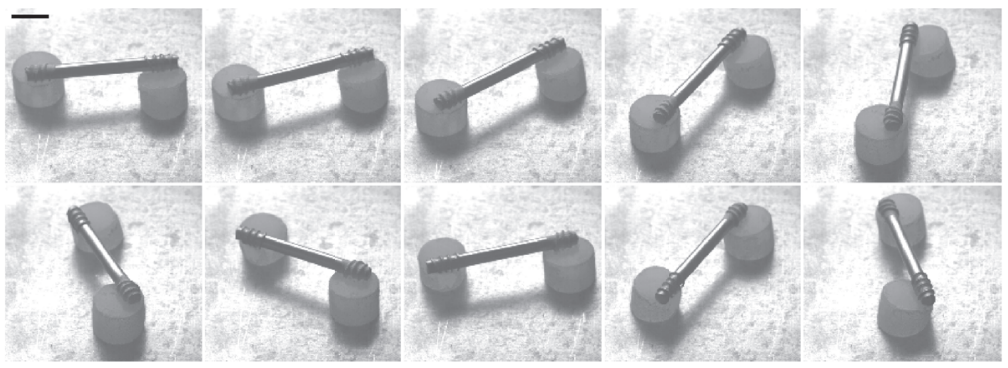

FIG. 6. Two platelets of dry ice linked by an off-centered steel rod rotate. Two successive pictures are separated by $200 \mathrm{~ms}$ and the bar shows $2 \mathrm{~cm}$. The angular acceleration deduced form these pictures is $2.0 \pm 0.1 \mathrm{rad} / \mathrm{s}^{2}$ (enhanced online). [URL: http://dx.doi.org/10.1063/1.4807007.5]

slope 1 in this log-log plot) quantitatively agreeing with the experiment. This agreement (established in the limit $m \ll M$ ) becomes logically less convincing as $m$ exceeds $2 \mathrm{~g}$, i.e., when it approaches $M$.

This model was developed for a static platelet. However, its velocity $v$ generates other flows (around, below), which might influence the motion. First, the platelet undergoes friction. Its origin can be either the shear in the vapor layer $\eta\left(v / h_{0}\right) R^{2}$ or the inertial friction $\rho R H v^{2}$ from the surrounding air of density $\rho$. Both forces balance the propelling force for a velocity of a few meters per second. In our experiments the velocity of the platelet never exceeds $10 \mathrm{~cm} / \mathrm{s}$ (Figure 4), so that we can neglect the friction in our measurement of the propelling force. Second, a well-known feature of the lubrication theory is that a tilted surface hovering over a horizontal wall creates a Poiseuille-Couette flow and thus undergoes a pressure force. ${ }^{13}$ In our case, the direction of propulsion in relation with the orientation of $\theta$ creates a depression: the resulting force $F_{P v}$ is normal to the bottom surface of the platelet and opposed to $F_{P}$. In the limit $\varepsilon \ll 1, F_{P v}$ scales as $\eta v R^{4} \theta / h_{0}^{3}$. For $v \approx 10 \mathrm{~cm} / \mathrm{s}, F_{P v}$ is on the order of $100 \mu \mathrm{N}$, that is, much smaller than $F_{P}: F_{P} \approx M g \approx 30 \mathrm{mN}$. This effect does not alter the propulsion either.

In comparison with a platelet on a ratchet, the propelling force obtained here is almost one order of magnitude higher. ${ }^{9}$ Moreover, the absence of texture in the surface prevents the platelet to sink and to get stuck: this system is much more efficient to propel Leidenfrost solids. Yet, the orientation of the force differs between both systems. With the ratchet, it is set by the substrate with the geometry of the textures, whereas the asymmetric platelet carries this orientation, which makes it change if the platelet rotates.

To consider the case of rotation, we study a device built such that the two coupled platelets are forced to move in opposite directions. As shown in Figure 6, the system consists of two cylinders of dry ice (mass $M=2.5 \mathrm{~g}$ ) linked by an off-centered steel rod of length $l=4.2 \mathrm{~cm}$ and mass $2 m=4.4 \mathrm{~g}$ that also acts as an added mass for the platelets. As seen in the figure, a rotation naturally results from this geometry. In addition, the motion accelerates as it does for the propulsion of single platelets. The angular acceleration in this example is $2.0 \mathrm{rad} / \mathrm{s}^{2}$. We expect an acceleration $2 F(m) /(m+M) l$, where the force $F$ is of order $200 \mu \mathrm{N}$ for an added mass per platelet of $2.2 \mathrm{~g}$, as seen in Figure 5(b). The resulting acceleration is thus $2.0 \mathrm{rad} / \mathrm{s}^{2}$, as observed experimentally.

The propulsion can also be seen with other solids, like camphor, as presented in Figure 7. Camphor's melting point is $179^{\circ} \mathrm{C}$, and its boiling point is $204^{\circ} \mathrm{C}$. Both temperatures are close, so that a piece of solid camphor put on a hot plate is surrounded by a small quantity of liquid camphor and the whole object levitates. Solid and liquid Leidenfrost effects here combine and the resulting object propels if a small mass is added on one side.

We showed that uneven platelets of dry ice can self-propel on a hot surface, and rotate when coupled together. The propulsion is due to the pressure exerted by the vapor flow on the bottom of the platelet. This surface is not parallel to the substrate but gets tilted by an angle $\theta$. The pressure generates a lateral force $M g \theta / 2$ creating a "continuous ratchet." This questions the evolution of the shape of the platelet while it sublimates. Since the thinner the vapor layer, the stronger the sublimation, the loss of matter is asymmetric below the platelet. Thus, the propelling force should be altered, which might be observed by following such objects on large distances. It would be also 


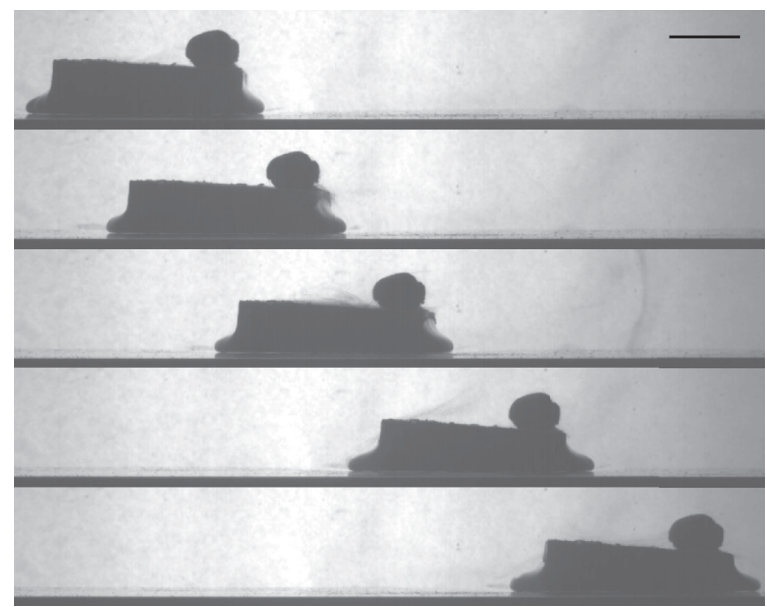

FIG. 7. A square platelet of camphor $(6.0 \mathrm{~g})$ with a small mass of $1.8 \mathrm{~g}$ on a hot plate $\left(400{ }^{\circ} \mathrm{C}\right)$. Although the solid is now surrounded by liquid camphor, the whole object still self-propels. Two pictures are separated by $0.65 \mathrm{~s}$ and the scale represents $1 \mathrm{~cm}$ (enhanced online). [URL: http://dx.doi.org/10.1063/1.4807007.6]

worth seeing how the propulsion is modified by the vapor flux: for instance, non-evaporative solids and liquids can be levitated by air flowing from the substrate with a velocity that does not depend on the thickness of the air layer. ${ }^{14}$ Finally, our results can be generalized to any Leidenfrost solid whose center of mass is not vertically aligned with the center of pressure on the bottom surface.

${ }^{1}$ J. G. Leidenfrost, De Aquae Communis Nonnullis Qualitatibus Tractatus (Ovenius, 1756).

2I. U. Vakarelski, J. O. Marston, D. Y. C. Chan, and S. T. Thoroddsen, "Drag reduction by Leidenfrost vapor layers," Phys. Rev. Lett. 106, 214501 (2011).

${ }^{3}$ T. Tran, H. J. J. Staat, A. Prosperetti, C. Sun, and D. Lohse, “Drop impact on superheated surfaces," Phys. Rev. Lett. 108, 036101 (2012).

${ }^{4}$ P. Brunet and J. H. Snoeijer, "Star-drops formed by periodic excitation and on an air cushion: A short review," Eur. Phys. J. Spec. Top. 192, 207-226 (2011).

${ }^{5}$ F. Celestini and G. Kirstetter, "Effect of an electric field on a Leidenfrost droplet," Soft Matter 8, 5992-5995 (2012).

${ }^{6}$ H. Linke, B. J. Alemán, L. D. Melling, M. J. Taormina, M. J. Francis, C. C. Dow-Hygelund, V. Narayanan, R. P. Taylor, and A. Stout, "Self-propelled Leidenfrost droplets," Phys. Rev. Lett. 96, 154502 (2006).

${ }^{7}$ G. Lagubeau, M. Le Merrer, C. Clanet, and D. Quéré, "Leidenfrost on a ratchet," Nat. Phys. 7, 395-398 (2011).

${ }^{8}$ A. Würger, "Leidenfrost gas ratchets driven by thermal creep," Phys. Rev. Lett. 107, 164502 (2011).

${ }^{9}$ G. Dupeux, M. Le Merrer, G. Lagubeau, C. Clanet, S. Hardt, and D. Quéré, "Viscous mechanism for Leidenfrost propulsion on a ratchet," Europhys. Lett. 96, 58001 (2011).

${ }^{10}$ T. R. Cousins, R. E. Goldstein, J. W. Jaworski, and A. I. Pesci, “A ratchet trap for Leidenfrost drops,” J. Fluid Mech. 696, 215-227 (2012).

${ }^{11}$ B. S. Gottfried, C. J. Lee, and K. J. Bell, "The Leidenfrost phenomenon: Film boiling of liquid droplets on a flat plate," Int. J. Heat Mass Transfer 9, 1167-1188 (1966).

${ }^{12}$ See supplementary material at http://dx.doi.org/10.1063/1.4807007 for the detailed development of Eq. (1).

${ }^{13}$ G. K. Batchelor, An Introduction to Fluid Dynamics (Cambridge University Press, Cambridge, 1967).

${ }^{14}$ M. A. Goldshtik, V. M. Khanin, and V. G. Ligai, "A liquid drop on an air cushion as an analogue of Leidenfrost boiling," J. Fluid Mech. 166, 1-20 (1986). 